\title{
An Easy Way to One-Dimensional Elastic Collisions
}

Jorge Sztrajman, Ciclo Básico Común, Universidad de Buenos Aires, Buenos Aires, Argentina Alejandro Sztrajman, University College London, London, United Kingdom

$\mathrm{T}$ The aim of this paper is to propose a method for solving head-on elastic collisions, without algebraic complications, to emphasize the use of the fundamental conservations laws. Head-on elastic collisions are treated in many physics textbooks as examples of conservation of momentum and kinetic energy:

$$
\begin{aligned}
& m v_{\mathrm{i}}+M V_{\mathrm{i}}=m v_{\mathrm{f}}+M V_{\mathrm{f}} \\
& \frac{1}{2} m v_{\mathrm{i}}^{2}+\frac{1}{2} M V_{\mathrm{i}}^{2}=\frac{1}{2} m v_{\mathrm{f}}^{2}+\frac{1}{2} M V_{\mathrm{f}}^{2} .
\end{aligned}
$$

$v_{\mathrm{i}}$ and $v_{\mathrm{f}}$ are the velocities before and after the collision for the mass $m$, and $V_{\mathrm{i}}$ and $V_{\mathrm{f}}$ are the velocities before and after the collision for the mass $M$. These equations are somewhat annoying to solve and produce the following final velocities: ${ }^{1}$

$$
\begin{aligned}
& v_{\mathrm{f}}=\frac{m-M}{m+M} v_{\mathrm{i}}+\frac{2 M}{m+M} V_{\mathrm{i}} \\
& V_{\mathrm{f}}=\frac{2 m}{m+M} v_{\mathrm{i}}+\frac{M-m}{m+M} V_{\mathrm{i}} .
\end{aligned}
$$

Other authors ${ }^{2}$ discuss the special case of one body initially at rest $\left(V_{\mathrm{i}}=0\right)$ to get a simplified version of Eqs. (3) and (4):

$$
\begin{aligned}
& v_{\mathrm{f}}=\frac{m-M}{m+M} v_{\mathrm{i}} \\
& V_{\mathrm{f}}=\frac{2 m}{m+M} v_{\mathrm{i}},
\end{aligned}
$$

or use the well-known anti-conservation of the relative velocities

$$
V_{\mathrm{f}}-v_{\mathrm{f}}=-\left(V_{\mathrm{i}}-v_{\mathrm{i}}\right)
$$

to work solely with linear equations. ${ }^{2}$

To contribute to a simple approach to these collisions, some proposals were made. Millet ${ }^{3}$ showed that both particles satisfy

$$
\begin{aligned}
& v_{\mathrm{f}}=2 v_{\mathrm{c}}-v_{\mathrm{i}} \\
& V_{\mathrm{f}}=2 v_{\mathrm{c}}-V_{\mathrm{i}},
\end{aligned}
$$

where $v_{c}$ is the velocity of the center of mass, and that this simple relationship can be used to make easy calculations. Later, $\mathrm{Hu}^{4}$ generalized this approach for non-elastic collisions and Funk ${ }^{5}$ pointed out the convenience of working in a frame where one of the bodies is at rest. These approaches intend to utilize easy-to-remember equations. However, it is common for students to solve this type of problem by mere manipulation of the equations, which does not lead to a deep understanding of underlying concepts. ${ }^{6}$

The approach we propose here is to move to the center of mass frame, where total momentum is zero. This is a strategy that has been commonly used in particle physics to study the production of new particles in collisional processes. The reason to choose such a frame is that in the collision of a particle with a system of bonded particles, only the energy in the center of mass frame is available for breaking the bond. ${ }^{7}$ We will show that this approach presents some conceptual and algebraic advantages, and can be translated to simple collision problems in the classroom. Center of mass velocity is given by

$$
v_{\mathrm{c}}=\frac{m v_{\mathrm{i}}+M V_{\mathrm{i}}}{m+M} \text {. }
$$

It should be emphasized that conservation of kinetic energy alone cannot guarantee that the collision is elastic. Why? Because conservation of kinetic energy is not a Galilean invariant; that is, it can hold relative to one inertial frame without holding relative to other inertial frames. However, if the momentum is also conserved, then the kinetic energy is conserved in all inertial frames if it is conserved in any one inertial frame. ${ }^{8,9}$ In our case, the conservation of momentum is a fundamental law and it holds in all inertial frames; so conservation of kinetic energy in the center of mass frame implies its conservation in all inertial frames.

In the center of mass frame, Eq. (1) is (we use "cm" to indicate velocities in the center of mass frame)

$$
m v_{\mathrm{i}}^{\mathrm{CM}}+M V_{\mathrm{i}}^{\mathrm{CM}}=m v_{\mathrm{f}}^{\mathrm{CM}}+M V_{\mathrm{f}}^{\mathrm{CM}}=0 .
$$

A simple inspection of this equation shows that there are two obvious solutions. One of them is $v_{\mathrm{f}}{ }^{\mathrm{CM}}=v_{\mathrm{i}}{ }^{\mathrm{CM}}$ and $V_{\mathrm{f}}^{\mathrm{CM}}=V_{\mathrm{i}}^{\mathrm{CM}}$, which means that the collision does not occur. The other is $v_{\mathrm{f}}^{\mathrm{CM}}=-v_{\mathrm{i}}^{\mathrm{CM}}$ and $V_{\mathrm{f}}^{\mathrm{CM}}=-V_{\mathrm{i}}^{\mathrm{CM}}$, and corresponds to the collision. Note that this change in the sign of the velocities does not alter the kinetic energy, and then Eq. (2) is satisfied. Thus, in that system, the collision is very simple: each mass bounces back as if it had hit a perfectly rigid wall (Fig. 1). ${ }^{10}$ Finally we return to the laboratory frame by adding the velocity of center of mass.

Hence, the steps of our approach are:

1. First we calculate the velocity of center of mass $v_{\mathrm{c}}$ given by Eq. (10).

2. Then, we obtain the initial velocity of each body in the new frame: 


$$
\begin{aligned}
& v_{\mathrm{i}}^{\mathrm{CM}}=v_{\mathrm{i}}-v_{\mathrm{c}} \\
& V_{\mathrm{i}}^{\mathrm{CM}}=V_{\mathrm{i}}-v_{\mathrm{c}} .
\end{aligned}
$$

3. The final velocities in that frame are simply the reversal of the initial velocities:

$$
\begin{aligned}
& v_{\mathrm{f}}{ }^{\mathrm{CM}}=-v_{\mathrm{i}}^{\mathrm{CM}} \\
& V_{\mathrm{f}}{ }^{\mathrm{CM}}=-V_{\mathrm{i}}^{\mathrm{CM}} .
\end{aligned}
$$

4. Finally, we go back to the laboratory frame by adding the velocity of the center of mass:

$$
\begin{aligned}
& v_{\mathrm{f}}=v_{\mathrm{f}}^{\mathrm{CM}}+v_{\mathrm{c}} \\
& V_{\mathrm{f}}=V_{\mathrm{f}}^{\mathrm{CM}}+v_{\mathrm{c}} .
\end{aligned}
$$

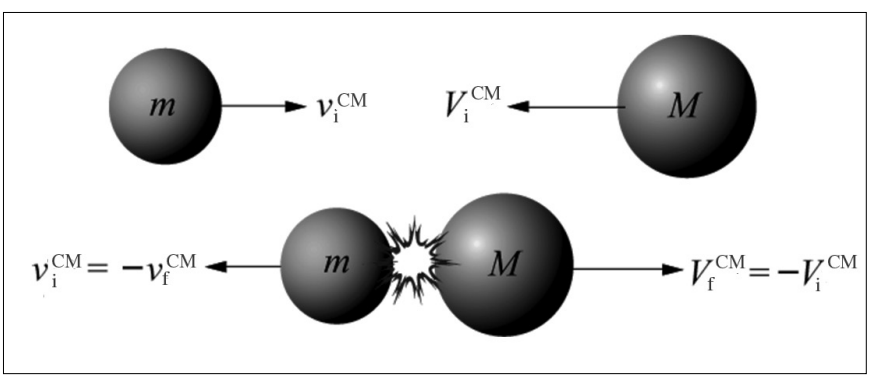

Fig. 1. From the center of mass frame, both bodies simply invert their velocities after colliding elastically.

Example (we use here the example of Ref. 5): A 5-kg car traveling at $6 \mathrm{~m} / \mathrm{s}$ east strikes a 3-kg car traveling at $9 \mathrm{~m} / \mathrm{s}$ west. If the collision is elastic, find the velocity of each car after the collision. In a frame fixed to Earth in which east is positive and west is negative, we have:

$$
\begin{aligned}
& m_{1}=5 \mathrm{~kg}, \\
& v_{1}=6 \mathrm{~m} / \mathrm{s}, \\
& m_{2}=3 \mathrm{~kg}, \text { and } \\
& v_{2}=-9 \mathrm{~m} / \mathrm{s} .
\end{aligned}
$$

The velocity of center of mass is:

$$
v_{\mathrm{c}}=\frac{5.6+3 .(-9)}{5+3} \mathrm{~m} / \mathrm{s}=0.375 \mathrm{~m} / \mathrm{s} .
$$

The initial velocities in the center of mass frame are:

$$
\begin{aligned}
& v_{\mathrm{i}}^{\mathrm{CM}}=v_{\mathrm{i}}-v_{\mathrm{c}}=6 \mathrm{~m} / \mathrm{s}-0.375=5.625 \mathrm{~m} / \mathrm{s} \\
& V_{\mathrm{i}}^{\mathrm{CM}}=V_{\mathrm{i}}-v_{\mathrm{c}}=-9 \mathrm{~m} / \mathrm{s}-0.375=-9.375 \mathrm{~m} / \mathrm{s},
\end{aligned}
$$

and the final velocities in the center of mass frame are:

$$
\begin{aligned}
& v_{\mathrm{f}}^{\mathrm{CM}}=-v_{\mathrm{i}}^{\mathrm{CM}}=-5.625 \mathrm{~m} / \mathrm{s} . \\
& V_{\mathrm{f}}^{\mathrm{CM}}=-V_{\mathrm{i}}^{\mathrm{CM}}=9.375 \mathrm{~m} / \mathrm{s} .
\end{aligned}
$$

Thus th final velocities in the frame fixed to Earth are:

$$
\begin{aligned}
& v_{\mathrm{f}}=v_{\mathrm{f}}^{\mathrm{CM}}+v_{\mathrm{c}}=(-5.625+0.375) \mathrm{m} / \mathrm{s}=-5.25 \mathrm{~m} / \mathrm{s} \\
& V_{\mathrm{f}}=V_{\mathrm{f}}^{\mathrm{CM}}+v_{\mathrm{c}}=(9.375+0.375) \mathrm{m} / \mathrm{s}=9.75 \mathrm{~m} / \mathrm{s} .
\end{aligned}
$$

\section{Conclusions}

Working in the center of mass frame, conservation laws result in a simple reversal of velocities. To go back to the laboratory frame and get the final velocities, we simply need to add the velocity of the center of mass. We hope that this procedure will help to focus the teaching and learning in concepts rather than calculations.

\section{Acknowledgment}

This work was partially supported by Universidad de Buenos Aires, Research Project, UBACyT, 20020130100390BA.

\section{References}

1. D. Halliday, J. Walker and R. Resnick, Fundamentals of Physics Extended, 10th ed. (Wiley, New York, 1997), p. 239.

2. H. D. Young and R. A. Freedman, Sears and Zemansky's University Physics, with Modern Physics, 14th ed. (Pearson Education, 2015), p. 252.

3. L. Edward Millet, "The one-dimensional elastic-collision equation: $v_{\mathrm{f}}=2 v_{\mathrm{c}}-v_{\mathrm{i}}$," Phys. Teach. 36, 186 (March 1998).

4. Hui Hu, "More on one-dimensional collisions," Phys. Teach. 40, 72 (Feb. 2002).

5. Deb Funk, "Elastic collisions with two moving objects made simple," Phys. Teach. 44, 80-81 (Feb. 2006).

6. Robert J. Dufresne, William J. Gerace, and William J. Leonard, "Solving physics problems with multiple representations," Phys. Teach. 35, 270-275 (May 1997).

7. Eugene Levin, "Energy in the center of mass," Am. J. Phys. 55, 909 (Oct. 1987).

8. T. L. Chow, Classical Mechanics, 2nd ed. (CRC Press, New York, 2013), p. 313.

9. D. K. Jha, Text Book of Vector Dynamics (Discovery Publishing House, New Delhi, 2005), p.107.

10. C. T. Tindle, "An intuitive approach to collisions," Phys. Teach. 36, 344-346 (Sept. 1998), and A. P. French, "Intuitively obvious?" Phys. Teach. 36, 453 (Nov. 1998).

Jorge Sztrajman is professor at the University of Buenos Aires. He received his $P h D$ in quantum gravity and his area of research is the didactics of physics.

jsztraj@gmail.com

Alejandro Sztrajman graduated from the University of Buenos Aires with an MSc in physics. He is currently a research assistant in the Virtual Environments and Computer Graphics group at University College London. a.sztrajman@ucl.ac.uk 